\author{
PETER MACHAMER \\ University of Pittsburgh \\ pkmach@pitt.edu \\ ALISON SPRINGLE \\ University of Pittsburgh \\ HPS \& Philosophy \\ alisonspringle@gmail.com
}

\title{
PERCEIVING AND KNOWING AS ACTIVITIES
}

\begin{abstract}
According to the tradition of most empiricists, perception is the basis for all our knowledge (at least of the world). The tradition also assumes that perception by humans is a passive activity resulting in some static states pertaining perception and belief, which are then, in some versions, modified by the mind before being passed onto memory and knowledge. Following the work of J. J. Gibson, we argue that perceiving involves many activities and actions. This is true of both visual as well as olfactory-taste perception. The main moral of this paper is that perceiving and knowing are best thought of not as involving static states, but rather as ongoing temporal activities involving change. This presumably means giving up a frozen ontology of states and moving towards something like a dynamic ontology as a basis.
\end{abstract}

Keywords. Activity, action, knowing, knowledge, perceiving, perception, dynamic ontology.

\section{Introduction}

First, a note: The title uses "activities" rather than "action", since "action" in its most usual philosophical use entails intentional behavior.

According to the tradition of most empiricists, perception is the basis for all our knowledge (at least of the external world). The tradition also assumes that perception by humans is a passive activity resulting in some static perceptual or belief states that then, on some versions, the mind modifies before passing the content onto memory and knowledge.

Following the work of J. J. Gibson, we argue that perceiving involves many activities and actions. This is true both of visual and of olfactory-taste perception.

More unusually, we also argue that knowing is an activity, again in many ways. (This follows a line of argument used by John Dewey, the American Pragmatist). Clearly, coming to know something (learning) involves many 
activities. Moreover, knowing itself is not just a static state or memory engram. Knowing is being able to draw inferences, solve problems and demonstrate the knowledge. Moreover, memory recall itself is most often a constructive activity (sometimes quite fanciful as in confabulation).

So the main moral in this paper is that perceiving and knowing are best thought about not as relations involving static states, but rather as ongoing temporal activities involving changes.

This presumably means giving up an ontology of static states and moving towards something like a dynamic ontology as basic.

\section{Perceiving}

We discuss three broad ways in which activity is involved in perception, using examples from visual and taste perception for each. We then discuss how the involvement of activity suggests that a dynamic picture of perception is more appropriate than static one. This dynamic theory derives in large part from the work of the psychologist J. J. Gibson (1904-1979).In emphasizing this active nature we do not mean to imply that all perception is perception for action. We enjoy as much as anybody reclining on a beach at sunset, sipping dark rum and lime juice while visually basking in the beauty of the moment. What we do wish to emphasize is that before Gibson, most if not all, work in perception dealt with object recognition which implies that only objects and their properties are important. This is probably a hangover from a substantialist ontological prejudice and it is this general position we wish to combat and argue against.

\subsection{Activity structures perception (telos)}

One of J. J. Gibson's (and the pragmatists that preceded him) key insights was that much of perception is preparation for action. Or in a slogan: Perceiving for a purpose (goal directed perception). Now this "for action" could mean many different things, and has meant many different things for different thinkers. So before getting to our examples, we will take a brief detour to discuss some of the things philosophers have meant by it. We will briefly touch on some of the controversies, abstaining from taking a position on these debates for the time being.

The least controversial version of the claim is probably this: a perceptual system's adaptive success - the reason it was naturally selected - is linked to how successfully it guided an organism's behavior in the environment in which an organism evolved. This is compatible with many diverse theories of perception. It is an informative claim to the extent that some philosophers, and, 
once upon a time, some psychologists, were inclined to treat perception in more intellectual and theoretical terms, rather than as a practical ability. That is, perception was taken to be a way of knowing (propositionally) about worldly objects and properties and their relationships to each other. The history of the philosophy of perception is tightly wound with the histories of epistemology and ontology. Both a cause and a consequence has been a sort of dualist assumption about the relationship between the mind and the world. The passive mind receives inputs from the world. The mind then transforms these inputs to greater or lesser degrees (depending on which philosopher you're asking) producing the mental representations of the world we have in perception (this position is often called "Constructivism"). The interface between the mind and the visual world is the eye, or more specifically, the retina. The world as projected onto the retina is not the world we perceive. So it's generally thought that the mind fills in the gaps, drawing a boundary between the world as we perceive it and the world as it really is, thus creating an epistemological puzzle about how perception can give us knowledge of the external world (if indeed it does). On this picture, the retina is like a camera film and the mind a sort of screen editor that interprets and adjusts shapes and colors of the film from the retina, and the resulting image constitutes the mind's access to the world.

The fairly uncontroversial insight that served to reorient our thinking about perception is that often perception is for action in that ultimately we perceive what and how we do because this helps us survive and reproduce in the environments in which our species evolved .The epistemological question: "is the world we perceive the way the world really is?" or, in other words, "what is the direct object of perception?" can come apart from the independently interesting metaphysical and empirical question: "what are the senses and perception good for - what do they do? How do they help creatures like this to survive in an environment like that?" or "the creature is surviving, so what's it doing such that the way it perceives is working so well for it?" Starting with the second question has led many psychologists and philosophers to better appreciate the importance of the organism's activity (the "enactive" component), of its body (the embodied component), and of its environment (the embedded component) to understanding perception and cognition both in human and nonhuman animals. ${ }^{1}$

\footnotetext{
${ }^{1}$ While these questions are conceptually distinct, many philosophers (and psychologists for that matter) still take them together, and they can certainly constrain one another (the epistemology of perception should respect the best science, but the plausibility of some of the theoretical assumptions or consequences of the science of perception depends on how sound they turn out to be in the realm of epistemology), Gibson, as well as many if not most of his followers, was a direct-realist, and this was an explicit part of his program. But as we explain, it's possible to be an indirect realist or even a transcendental idealist and take on board much of the enactive, embodied, embedded program.
} 
It should be noted that this fairly uncontroversial insight is compatible even with representationalism, which Gibson, who denied representations, and many contemporary neo-Gibsonians and similar embodied, embedded, enactive, and extended views, would consider in opposition with his program. ${ }^{2}$ It's compatible insofar as biological function, which is understood essentially as we have defined the uncontroversial version of the claim that perception is for action above, plays a constitutive role in a number of representational accounts (e.g. Dretske, Papineau, Millikan, \& O'Shea). ${ }^{3}$

One place controversy turns up is in the implications of perception being for action. For instance, a representationlist might argue that all we perceive are properties, but the properties we represent are represented because of their biological usefulness for action. It would thus be open to one to maintain the dualism between mind and world. Perception is for action, but the information perception delivers to guide action is impoverished and requires interpolation by mental processes. Alternatively, a Gibsonian might argue that perception (perceptual content, where this need not imply representations) is much thicker. That in perception already - not just when cognition has come into play - we are presented immediately with practically relevant information, such as the functional significance of things in the environment and the opportunities for behavior that they allow, or what Gibson termed "affordances." Perception needs to tell us what's dangerous, what's food, what's a mate, where one might hide, etc. and there may be resources in an organism's environment (including other organisms) that directly inform the organism of these facts.

Even if one does not go in for the direct perception of affordances, taking seriously the idea that perception is for action introduces some constraints on one's theory of perception. For instance, if we perceive so that we may react to a predator, then perception had better be fast enough for the organism, typically, to avoid being killed. So, inefficient, time consuming processing cannot be what's involved in initiating action to avoid a predator or other impending danger, as demonstrated by time to collision experiments where various species of animals demonstrated avoidance behavior when faced with an apparently impending impact from an approaching object. ${ }^{4}$ There cannot, for instance, be something like a deliberative, practical inference taking place (that's not to say there may not be something very fast and inference-like occurring, but it must be able to work very quickly), because cognitive processing would seemingly take

\footnotetext{
${ }^{2}$ For review and defense of embodied, embedded, enactive and extended views, see WARD and STAPLETON [2012].

${ }^{3}$ See e.g. DRETSKE [1995] and [1988], PAPINEAU [1984], [1987] and [1993], MILLIKAN [1984], [1989a] and [1989 b] and O'SHEA [2013].

${ }^{4}$ SCHIFF [1965].
} 
too much time. Further, the visual systems in simple creatures are capable of using this information - of moving directly from the perception of the world to an appropriate activity with respect to it - but it is doubtful that they are performing inferences. Similarly, it cannot be that in order to perceive a predator or danger, or to perceive something as dangerous, e.g. infants and the visual cliff, and Schiff collision avoidance experiments ${ }^{5}$, one must first have some higher-level conceptual understanding of what a predator is, since presumably infants, sheep and bunnies, for instance, lack such concepts, but are able to dodge predators when the need arrives, presumably on the basis of their perceiving them. Where perceptual systems can quickly, reliably, and directly tell the organism how to react appropriately in its environment, there's reason to think they will do so - that in some cases they have been adapted to do so. So we think the fact that perception is for action (activity) provides a heuristic for theorizing about perception: perceptual systems and perceptual content have a very tight relationship to activity, and with respect to that relationship, evolution favors efficiency and economy. This doesn't yet provide an answer to exactly what the relationship is (e.g. causal vs. constitutive), but provides what we consider an underappreciated constraint (though not by all, arguably Millikan's pushmi-pullyu representations were inspired in part by such considerations) which may help decide some conflicts. ${ }^{6}$

We will now provide a few fairly straightforward examples of ways in which perception is for action, again, focusing on vision and gustation.

One of the functions of vision is to let us know how to respond appropriately, e.g. to food, predators, mates, obstacles to our movement, and other objects that we may be desiring or avoiding. This appears to be a function of foveal vision. Foveal (as opposed to peripheral) vision has high acuity and so provides information needed to identify specific objects by e.g. color, and shape. When we need to identify an object, to see what kind of object it is, we actively bring it into focus by foveating on it. We, usually, identify objects, of course, so that we may do (the appropriate) things with them - this is presumably the raison d'etre of our recognitional capacities. For instance, we perceive their shapes and distances from us so that we may know if and how we may grasp them - i.e. what size to make our grip aperture and how to grip the object (if it is slippery we will grasp it one way, if it is rough or prickly, quite another. Of course, tactile perception also plays an important role here, but we shall continue to focus on vision). Alternatively, we perceive and identify objects so that we may plan actions involving them.

\footnotetext{
${ }^{5}$ On visual cliff, see: GIBSON, PICK [2000] and GIBSPON, WALK, TIGHE [1957]. On time to collision see: SCHIFF, DETWILER [1979] and GIBSON [1969].

${ }^{6}$ MILLIKAN [1996].
} 
Peripheral vision, in contrast, doesn't tell us much (though not nothing) about what objects are where in our environment; it is very low in acuity and achromatic. But it does detect motion and, thus, change. Peripheral vision tells us e.g. when something is approaching us, or when something small has scampered by. Peripheral vision tells us when to turn our heads so that we may foveate on and identify what is coming our way and what has dashed by. So peripheral vision directly informs activity in the form of head and body movement, movement in the direction of an object on which we will foveate, in order to identify, so that we may act on or respond to it appropriately.

Turning now to gustation: thinking of gustation (that is, the taste and flavor sensory system) in terms of its ecological (and thus social and biological) function has implications for how one takes the gustatory system to be organized. If one thinks of gustatory perception in terms of the reception of information from the environment, specifically from certain chemicals in the environment, one might be inclined to consider taste only in terms of certain receptors, that is, sites sensitive to select stimuli. In fact, this is commonly done by psychologists, who identify our sense of taste with the receptors on the tongue and the roof of the mouth. ${ }^{7}$

In contrast, if you think of the sense of taste in terms of the biological and social functions of selecting food that is healthy for consuming (non-poisonous and nutritious) or socially significant, and follow Gibson in considering the sense of taste as a perceptual system, there's compelling reason to move beyond the tongue and roof of the mouth. In addition to these organs, the nose (for retronasal olfaction) and the trigeminal nerve (for somatosensation) play crucial roles in flavor perception. While the tongue is in part responsible for the sensation of sweetness, saltiness, bitterness, and sourness, retronasal olfaction is responsible for most of the flavor experiences we identify, e.g. vanilla, chocolate, coffee, etc. And somatosensation (both in the nose and in the mouth) is responsible for the sensation of viscosity (as from fat - e.g. cream and butter), spiciness (jalapeño), and mint flavor. Retronasal olfaction can also influence how sweet or sour something tastes, as can temperature. ${ }^{8}$ And in addition to somatosensation, audition plays a role in texture sensation e.g. crunchiness - indeed, potato chip companies select noisy, crinkly materials for bags so that the chips will seem fresher. ${ }^{9}$ The sound carries information about whether or not it should be eaten - if something is stale, for instance, then it's probably old and may possess dangerous bacteria or fungi. Olfaction tells us a lot about whether food has passed the point at which it is safe for consumption. ${ }^{10}$

\footnotetext{
${ }^{7}$ WOLF, KLUENDER, LEVI [2012], Chapter 15.

${ }^{8}$ See CRUZ, GREEN [2000].

${ }^{9}$ See e.g. WINTERMANN [2012].

${ }^{10}$ WOLF, KLUENDER, LEVI [2012].
} 
These different kinds of information work together to tell us about what foods we should eat (an activity of biological and social import). And they work together not only bypooling their findings, but by actually influencing each other - something that smells like vanilla (something with vanilla flavor) will be perceived as sweeter than something that smells like lettuce (this is the phenomenon of "sweetness enhancement?"). ${ }^{11}$

\subsection{Activity in information pickup}

The previous section discussed the teleological or functional role of perception with respect to action (activity). The claim was that perception is often for action (activity), and that this makes a difference to how we conceive of and theorize about perception. We now move onto a different feature of the relationship between perception and activity, namely, the causal or constitutive role of activity in the very act of perceiving. To wit, we've just discussed ways of understanding the fact that we perceive in order to act. We now discuss ways in which we are active so that we may perceive.

As was the case with the teleological or functional role of activity in perception, there are more and less controversial ways of understanding the role of activity in perception. The less controversial or more traditional way which is compatible with the static (eye as a camera) view is to understand the role of activity instrumentally. On this view, one acts so that one may be in a position to receive stimuli from the environment, but perception really begins after the stimuli have been received. One can thus maintain the kind of dualistic view mentioned earlier, and give an instrumental role to activity in perception.

However, Gibson held that once one appreciates the activity that's involved in perception, the idea that the eye is taking pictures of features in the environment that the mind then processes (strings together and interprets) to create representations of the external world loses force. This is because as one moves around, a great deal of information is revealed in the environment. According to Gibson, activity obviates the need to mentally construct a perceptual (e.g. visual) scene. One aspect of his critique of traditional psychological theories of perception dealt with their experimental designs. In order to make perception something that could be subjected to experimental testing, experimental paradigms had to use highly artificial situations (e.g. tachistoscopes), and were therefore devoid of a great deal of information that is provided in natural, ecological settings (the settings in which our perceptual systems evolved and normally function). In these experiments, subjects are typically instructed to stay still and to fixate on a single point or on several isolated points at a time, or on

11 See e.g. STEVENSEN, BOAKES [2004]. For philosophical discussion, see SPENCE, AUVRAY, SMITH [2015]. 
a simple object. As a result, psychologists came to believe that the information we receive from the retina is insufficient to specify visual features such as multidimensional shape and depth. Rather, what the visual system directly receives are "clues" or "cues" (e.g. pictorial depth cues) that perceptual processing then uses to interpret and re-construct the visual scene. Gibson held that psychology designed its experiments this way in part because it assumed that perception is in the business of taking snapshots of the world. Rejecting this picture of perception, Gibson (and others including Elanor Gibson, Anne Pick, and Egon Brunswik) found ways to perform experiments in natural, ecological settings. ${ }^{12}$ And in these settings, it seemed there was no need to mentally augment the incoming visual information. ${ }^{13}$

Gibson's radical view was that there's enough visual information available in the ambient array of reflected light for most tasks, so there is no need to construct mental representations of the world. This information is only available in a natural environment (for instance, the texture of the ground or floor is very important, as is the horizon) and the information is picked up by an organism as the organism moves about in that environment.

We will now move onto some examples:

J. J. Gibson served in WWII as a director of a unit for the U.S. Army Air Force's Aviation Psychology program. ${ }^{14}$ One of the fruits of his research in the Air Force was the discovery of Optic Flow. Optic flow is the pattern of change in the structure of visible light caused by the relative motion between an observer and an environment. It's these changes in the optic array that allow us to perceive invariants - structures that are resistant to the change brought about by our movement - and the invariants constitute the information about the environment that we need, e.g. when a pilot is coming in for a landing, the shape and location of the landing is specified by the invariant structures in the optic flow, and velocity with which the vessel is moving towards the landing will be specified by the rate and direction of the flow

In the next section we will discuss other ways in which activity may contribute to what is perceived.

Gustation perhaps provides more obvious examples of the crucial role activity plays in information pick-up. According to the oyster chefs interviewed by Bon Apetit's "How to Eat (and Taste) an Oyster," looking, smelling, and chewing is essential to detecting an oyster's true flavor. Similarly, as you'll learn at any wine tasting, a tasting ritual consists in: sip, air intake, and swish around in the mouth (especially the back of the mouth), followed by swallow or spit. Here we

\footnotetext{
${ }^{12}$ GIBSON [1969] and [1991] GIBSON, PICK [2000], BRUNSWIK [1934], [1943], and [1947].

${ }^{13}$ GIBSON [1986], Chapter 9.

${ }^{14}$ REED [1988].
} 
will discuss several of the many ways in which activities are essential to extracting information in gustation.

Gordon Shepherd, a professor of neurobiology, has devised a nice procedure for demonstrating how multi-modal taste perception is. He calls it the "nose-pinch test." ${ }^{\prime 15}$ Here it is: hold your nose tightly and take a sip of your coffee and swish it around in your mouth, or pop a mint or piece of gum into your mouth and chew it. While holding your nose, you'll taste very little - depending on what the substance is, you'll get sweet, salty, bitter, sour, and maybe some burning or tingling. This is because the information you're extracting is limited to what the taste buds on your tongue and the roof of your mouth can extract. But then let go of your nose and breathe in. You'll experience a wave of coffee or mint flavor.

Shepherd's test highlights the role of retronasal olfaction in extracting information about the food or drink we are eating. Retronasal olfaction is the nasal passage that connects up with the back of your mouth. Of course, as wine and scotch drinkers know well, retronasal olfaction is only part of the story. Orthonasal olfaction, specifically, active sniffing, extracts yet further information about the substance you're about to consume, or considering consuming.

When discussing Shepherd's test, we mentioned that while you were holding your nose, you might experience some burn or tingle, depending on the substance. For instance, if you're eating chili pepper, horseradish, or mint. Or perhaps you're experiencing the delectable viscosity of some buttery or creamy dessert. The trigeminal nerve (touch) contributes these features of experience.

The ears also extract information concerning the texture of the food we eat, as mentioned above.

These are just a few examples of the many ways different sensory modalities are involved in flavor experience. And notice that activity is involved in all of this - chewing, breathing, swallowing, etc. It may be that, as Matthew Fulkerson (2014) argues, the distinct receptors involved in touch (somatosensation) work together in the activities involved picking up information about objects resulting in a unified phenomenology of touch. We suggest that these activities which structure the act of eating may help explain the unified phenomenology of flavor. ${ }^{16}$ For instance, the fact that when eating pretzels the experience is as of a smooth, crunchy, salty object located in the mouth, and not discrete sensations from each of the involved modalities (ears, tongue, mouth, etc.).

\footnotetext{
${ }^{15}$ SHEPHERD [2012].

${ }^{16}$ FULKERSON [2014].
} 


\subsection{Activity structures perceptual experience (form)}

We have discussed the ways in which perception can be thought of as being for action (activity) and the ways in which activity can be thought to be important for perception. In this section, we discuss the ways in which activity figures into what is perceived.

On a traditional picture, activity simply doesn't figure into the content of what's perceived. Perception may be for action, and the functions of our perceptual systems, which on some traditional pictures play a role in determining content, may be related to behavior/activity on the part of the organism, but the behavior/activity has no place in the content itself.

One version of this static view is known as the "snapshot" theory of perception. According to this theory, (visual) perception is like a fast-acting camera, taking snapshot after snapshot, and then mentally stringing the snapshots together to form coherent scenes through space and time. As we move around in the world, it's just as if you are taking photographs from different angles or positions within the world, now of this object, now of that. Perception is just a series of static, momentary states.

Against this, Gibson asserted that perception is an event: it persists through time, it has duration. And one perceives the world not by standing still and snapping a perceptual picture, but by moving about in and acting on the world - e.g., walking around objects. Such activity is necessary to create access to information in the structure of the optic array. As Gibson explains:

\footnotetext{
The optic array changes, of course, as the point of observation moves. But it also does not change, not completely. Some features of the array do not persist and some do. The changes come from locomotion, and the nonchanges come from the rigid layout of the environmental surfaces. Hence, the nonchanges specify the layout and count as information about it; the changes specify locomotion and count as another kind of information, about the locomotion itself. $(73)^{17}$
}

Gibson distinguished between two kinds of structure in the optic array: invariant structure and perspective structure. Perspective structure "changes with every displacement of the point of observation - the shorter the displacement, the smaller the change, the longer the displacement, the larger the change." (73) The invariant structure remains the same regardless of motions of the observer and thus "separates off best when the frozen perspective structure begins to flow." Further, information from invariants (about surfaces in environment) implies information about perspective (position, locomotion), and vice versa.

\footnotetext{
${ }^{17}$ GIBSON [1986].
} 
For instance, objects uniformly "growing" or "shrinking" as an observer moves towards or away from them is an example of an optical flow invariant, as the array will always transform like this under those changes.

It's not that the information merely takes movement to be revealed, as if the movement were just needed to pull the curtain, making way for the perceptual systems to take a picture. Instead the act of moving, the dynamic inter-activity between the perceiver and the world, necessarily go together. As Gibson explains:

The geometrical habit of separating space from time and imagining sets of frozen forms in space is very strong. One can think of each point in the medium as stationary, and distinct to each such point there would correspond a unique optic array. The set of all points... and optic arrays is the whole of the available information about the layout...This is an elegant and abstract way of thinking, modeled on projective geometry. But it does not allow for the complexities of optical change and does not do justice to the fact that the optic array flows in time instead of going from one structure to another. What we need for the formulation of ecological optics are not the traditional notions of space and time but the concepts of variance and invariance considered as reciprocal to one another. (74 ibid)

It is important to realize that the flowing perspective structure and the underlying invariant structure are concurrent. They exist at the same time. Although they specify different things, locomotion through a rigid world in the first instance and the layout of that rigid world in the second instance, they are like the two sides of a coin, for each implies the other...there is nothing illogical about the idea of concurrent specification of two reciprocal things. (76 ibid.)

The environment seen-at-this-moment does not constitute the environment as it is seen. (195 ibid.)

Another way in which Gibson thought activity alters the very content of experience is in what he thought the content of experience was. Some traditional notions of perceptual or experiential content tend to be relatively thin. Organisms perceive colors, shapes, properties, and objects, e.g. the green leaves of a tree. But they do not see the leaves as leaves, or the tree as a tree, where this requires applications of concepts that only adult humans possess.

Gibson, in contrast, has a richer notion of the content (which he understood to be non-representational, though given the many different things "representation" might mean one must be cautious before assuming to know what he meant by this). According to Gibson, what organisms perceive are not, or at least not merely, objects and properties, but affordances; an affordance is what some object or situation allows an organism to do or not do, for example, a sharp drop in the plane on which the organism is moving affords falling (this situation is experimentally simulated by a visual cliff). The point is that seeing for Gibson 
is seeing as, where the "as" is construed as information relevant to possible behaviors for the organism, and these possibilities need not require the capacity for high-level conceptual (or linguistic) thinking. Creatures see the world as opportunities for behavior or as meaning or entailing bodily consequences.

We have just suggested that activity determines content in terms of its duration, dynamic as opposed to static, and in terms of what the content means for the organism - affordances as opposed to subject-independent descriptions of objects and properties. Other philosophers of broadly Gibsonian, pragmatist, or phenomenological leanings have posited further roles for activity in determining and even constituting content.

For instance, Alva Noë (2004) has argued that organisms gain a kind of knowledge-how, a knowledge or expectation of sensori-motor contingencies by actively moving around in and exploring their environments. ${ }^{18}$ According to Noë, to perceive an object such as an apple as having volume, one must bring to bear sensori-motor knowledge, e.g. grasping, about objects of that kind,

Another claim Noë makes is about occlusion and a-modal completion. Organisms are able to perceive objects and shapes as the whole objects and shapes they are even when they are partially occluded. Noë borrows Gibson's example of seeing a cat partially occluded by the rails of a picket fence. We perceive the cat as whole even though we are only directly visually confronted with cat slices. Saccadic eye movements are necessary to take in whole scenes or object, so even wholeness of object requires activity, which for Noë is largely proprioceptive. So for Noë, my visual perception as of a full object or scene includes in its content a kind of proprioceptive readiness to explore, via saccades, the visual scene, thereby making available information that is not currently visually available or presented.

We are not arguing in favor of Noë's views. In fact, we want to emphasize Gibson's notion of reciprocity in perception: every experience of the world also gives us egocentric proprioceptive information. It's not just that proprioception informs vision, vision informs proprioception. We thus reject views that attempt or seem to attempt to make proprioception more basic than vision. However, we do think Noë highlights a potentially important feature about the content of perception that we are inclined to endorse, i.e., that content is sometimes, or perhaps even often, multi-modal, and multi-modality requires and implies potential activity. Sensori-motor content is about seeing and expectations of how we might act. Noë is right that the senses that carry information about an organism's motor activity are somatosensory (e.g. muscle spindles), proprioceptive, and vestibular, but also, at times, visual.

${ }^{18}$ NOË [2004]. We are aware that expectation plays a role in many theories of perception and knowledge, e.g. the perceptual schemata of Julian Hochberg [1964], Roger Shank's [1990] scripts. 
A fairly simple example of the way in which visual content relies on and may be thought to include proprioceptive information is in detecting movement. Movement of light across the retina can either be caused by the movements of the eye or the movements of a light reflecting object. To perceive an object moving, the visual system must distinguish an object's movement from the eyes' movement. Some theories hold that proprioceptive information is merely used in the computations that result in the perception as of a moving object. However, it's also possible part of the content of our awareness is that the perceived movement is not ocular or bodily movement. To wit, the content might be "moving object" or it might be "movement not from the perceiver" which entails that the movement is of something external. As should be clear, we endorse something along the lines of the latter interpretation of the content.

Another way in which visual content may be thought to be multi-modal is in the perception of visual texture and hardness. When a surface is represented as rough, this is different from representing a surface as smooth or merely patterned in a certain way. This information is important to how we attempt to interact with the object - for instance, how we should walk on that surface. Visual roughness seems to have direct tactile significance. The incorporation of tactical content in visual experience parallels the modal integration of information characteristic of flavor experience discussed above.

\section{Knowing}

As noted in the introduction, most epistemic theories, notably empiricism, hold that our knowledge of the world somehow derives from the content of our perceptions of the world. An active view of perception and knowledge does not deny this.

Rather, the active theory of perception holds that we get knowledge from perceiving, but often it is knowledge about how to act. (And so it is knowledge not in propositional form). It is, also, information about the layout of the world. In this way active perceiving is the basis for active knowing. How and why knowing is active may be explained in many ways.

Perhaps, the easiest way to conceive of the active nature of knowing is to contrast it with a passive view (as we saw earlier in perception). A passive state theory holds that knowledge consists in holding true beliefs, where holding or entertaining a belief is just for a person or mind to be in a stable relation to a proposition. This makes all knowledge propositional knowledge. 
Now, commonly memory psychologists distinguish different kinds of memory. The memory system encoding propositions is called declarative or semantic memory, but then they add other memory systems, i.e. episodic memory, which is the system that encodes episodes in a person's life so there is always a reference to the person's ego or self, and finally, there is procedural memory, which is knowing how to do something. The first two systems are often conceived as having propositional content.

A few philosophers, e.g. Jason Stanley (2011), believe and try to argue that even knowing how is propositional, but we won't enter this dispute here.

But let's think about thinking (as John Dewey might say). When we think we seldom confine ourselves to one sentence-like thought, even if we think in words. Our thinking often races from thought to thought, drawing inferences, solving problems or constructing fantasies. That is, we actively use our knowledge when planning to do something.

So, e.g. when we move to get information about the shape and distance of an object, it might well be that we obtain this information or knowledge in order to grasp or lay hold of an object. Or, e.g., use the knowledge gained from the seeing of an object coming toward us to avoid collision with that object. ${ }^{19}$

But there are other less obvious ways to think about the active character of knowing: Most generally, knowing is an adaptation of humans interacting with their environment. And a person can come to know something more or less well. Knowing is not an all or nothing condition. We have to do things to gain more knowledge. Obviously learning and gaining knowledge is an active process.

But more about the active nature of knowing itself. First, a long-standing, fairly popular analytic tradition holds that knowledge is justified true belief. Now an influential group of philosophers hold the position that justification is comprised by the giving of reasons. And reasons must be made explicit and public by language. These arguments that follow should not be confused with any form of linguistic behaviorism (even in a Wittgensteinian or Rylian form).

Yet another argument for the active nature of knowledge is that for any knowledge to be meaningful, it must be put to use, or, phrased differently, knowing must have consequences or effects, for the knower and/or for others. This would be to conceive of knowledge as dispositional. It must be capable of being actualized to count as knowledge. Actualization or consequences may come in many ways, e.g. as answers to questions, as bases for inferences, or as steps in problem solving, or even as constructing content for daydreams or fantasies by thinking.

${ }^{19}$ See SCHIFF, DETWILER [1979]. 


\section{References}

Bon Appétit, [2013], "How to Eat (and Taste) an Oyster", Bon Appétit, Feb 12 http://www.bonappetit.com/trends/article/how-to-eat-and-taste-an-oyster.

BRUNSWIK, E. [1934], Wahrnehmung und Gegenstandswelt: Grundlegung einer Psychologie vom Gegenstand her. Leipzig: Deuticke.

BRUNSWIK, E. [1943], Organismic Achievement and Environmental Probability. Psychological Review 50: 255-272.

BRUNSWIK, E. [1947], Perception and the Representative Design of Psychological Experiments. 2 d ed., rev. \& enl. Berkeley: Univ. of Calif. Press.

CRUZ, A., GREEN, B. G. [2000], “Thermal Stimulation of Taste”, Nature 403, pp. 889-892.

DRETSKE, F. [1988], Explaining Behavior: Reasons in a World of Causes, Cambridge, Mass. The MIT Press.

DRETSKE, F. [1995], Naturalizing the Mind, Cambridge, Mass.: The MIT Press.

FULKERSON, M. [2014], The First Sense: A Philosophical Study of Human Touch MIT Press.

GIBSON, E. J. [1969], Principles of perceptual learning and development. New York: Appleton-Century-Crofts.

GIBSON, E. J. PICK, A. D. [2000], An ecological approach to perceptual learning and development. New York: Oxford University Press.

GIBSON, E. J. PICK, A. D. [1991], An odyssey in learning and perception. Cambridge: MIT Press.

GIBSPON, E. J., WALK, R. D., TIGHE, T. J. [1957], "Behavior of Light- and Dark-Reared Rats on a Visual Cliff'. Science 126 (3263): 80.

GIBSON, J. J. [1986], The Ecological Approach to Visual Perception, Psychology Pres Taylor \& Francis Group.

HOCHBERG, J. [1964], Perception, Prentice Hall, Incorporated.

MILlIKAN, R. [1984], Language, Thought and Other Biological Categories, Cambridge, MA: MIT Press.

MILLIKAN, R. [1989a], “In Defense of Proper Functions”, Philosophy of Science, 56, no. 2: 288302, and reprinted in Millikan, 1993(a) op. cit.

MILLIKAN, R. [1989b], “Biosemantics”, Journal of Philosophy, 86: 281-97.

MILlIKAN, R. [1996], "Pushmi-Pullyu Representations”, [in:] J. Tomberlin (ed.), Philosophical Perspectives, Vol. 9, Atascadero, CA: Ridgeview Publishing, 185-200.

NOË, A. [2004], Action in Perception, MIT Press.

O’SHEA, N. [2013], "Naturalising Representational Content”, Philosophy Compass, 8 (5), pp. 496-509.

PAPINEAU, D. [1984], "Representation and Explanation", [in:] Philosophy of Science, 51: $550-572$. 
PAPINEAU, D. [1987], Reality and Representation, Blackwell Pub.

PAPINEAU, D. [1993], Philosophical Naturalism, Blackwell Pub.

REED, E. S. [1988], James J. Gibson and the Psychology of Perception, New Have: Yale University Press.

SHANK, R. [1990], Tell Me a Story: Narrative and Intelligence, Northwestern University Press.

SCHIFF, W. [1965], "Perception of impending collision: A study of visually directed avoidant behavior." Psychological Monographs: General and Applied, vol. 79(11), pp. 1-26.

SCHIFF, W., DETWILER, M. L. [1979], "Information Used in Judging Impending Collision" Perception, 8, pp. 647-58.

SHEPHERD, G. [2012], Neurogastromony: How the Brain Creates Flavor and Why It Matters Columbia University Press.

SPENCE, C., AUVRAY, M., SMITH, B. [2015], "Confusing Tastes with Flavors", [in:] Dustin Stokes, Mohan Matthen, and Stephen Biggs (eds), Perception and Its Modalities, Oxford University Press, pp. 247-274.

STANLEY, J. [2011], Know How, Oxford University Press.

STEVENSEN, R. J., BOAKES, R. A. [2004], "Sweet and Sour Smells: Learned Synesthesia Between the Senses of Taste and Smell", [in:] G. A. Calvert, C. Spence, \& B. E. Stein (eds), The Handbook of Multisensory Processing, (pp. 69-83), Cambridge, MA: MIT Press.

WARD, D., STAPELTON, M. [2012], "Es are good: Cognition as Enacted, Embodied, Embedded, Affective and Extended", Consciousness in Interaction: The Role of the Natural and Social Environment in Shaping Consciousness. Fabio Paglieri. John Benjamins (eds), pp. 89-104 (Advances in Consciousness Research, No. 86).

WINTERMANN, D. [2012], “Future Foods: What will we be eating in 20 year's time?”, [in:] BBC News Magazine, July 30 http://www.bbc.com/news/magazine-18813075.

WOLF, J., KLUENDER, K., LEVI, D. [2012], Sensation \& Perception Third Edition, Sinauer Associations Inc. 